Proceedings

\title{
Synthesis and Piezoelectric Characterization of UV- Curable Nanocellulose/ZnO/AlN Polymeric Flexible Films for Green Energy Generation Applications ${ }^{\dagger}$
}

\author{
Maria Assunta Signore ${ }^{1}$, Giulio Malucelli ${ }^{2}$, Donatella Duraccio ${ }^{3, *}$, Chiara De Pascali ${ }^{1}$, \\ Ambra Fioravanti ${ }^{4}$, Pietro Siciliano ${ }^{1}$ and Luca Francioso ${ }^{1, *}$ \\ 1 Institute for Microelectronics and Microsystems-CNR-IMM, Via Monteroni, 73100 Lecce, Italy; \\ mariaassunta.signore@cnr.it (M.A.S.); depascali@le.imm.cnr.it (C.D.P.); pietro.siciliano@le.imm.cnr.it (P.S.) \\ 2 Department of Applied Science and Technology, Politecnico di Torino, Viale Teresa Michel 5, \\ 15121 Alessandria, Italy; giulio.malucelli@polito.it \\ 3 Institute of Sciences and Technologies for Sustainable Energy and Mobility-CNR-STEMS, \\ Strada delle cacce 73, 10135 Torino, Italy \\ 4 Institute of Sciences and Technologies for Sustainable Energy and Mobility-CNR-STEMS, \\ Via Canal Bianco 28, 44124 Ferrara, Italy; a.fioravanti@imamoter.cnr.it \\ * Correspondence: donatella.duraccio@stems.cnr.it (D.D.); lucanunzio.francioso@cnr.it (L.F.); \\ Tel.: +39(0)11-3977-617 (D.D.); +39(0)83-2422-525 (L.F.) \\ + Presented at the 4th International Conference nanoFIS 2020-Functional Integrated nano Systems, \\ Graz, Austria, 2-4 November 2020.
}

Published: 30 December 2020

\begin{abstract}
In this work, the fabrication of composites consisting of piezoelectric $\mathrm{ZnO}$ ceramic nanostructures and nanocellulose fillers in a UV-cured acrylic matrix has been exploited for the design of new functional coatings for green energy generation. The piezoelectric behavior was investigated at different accelerations applied to cantilever beams. The piezoelectric signal generated by the different $\mathrm{ZnO}$ nanostructures was improved by aluminum nitride film integration on the beam and proof mass insertion at the tip.
\end{abstract}

Keywords: flexible composites; sol-gel methods; piezoelectric characterization

\section{Introduction}

Energy harvesting with piezoelectric materials has attracted interest in the scientific community [1]. They have the advantage of being easily deposited on flexible substrates, a mandatory feature for innovative devices in the field of green technologies. Composites consisting of piezoelectric ceramic fillers embedded in a flexible polymeric matrix show higher flexibility, simple integration into devices, size reduction, reliability improvement of devices, and lower production costs. In this context, the combination of nanocellulose and zinc oxide $(\mathrm{ZnO})$ nanostructures has been considered as a very promising method for fabricating self-powered nanogenerators.

\section{Materials and Methods}

$\mathrm{ZnO}$ flowers or needles nanostructures, synthesized by aqueous sol-gel approach, were dispersed together with cellulose nanocrystals in a UV-curable acrylic system (Ebecryl 150) and used as piezoelectric fillers at $4 \mathrm{wt}$ \% loading. The samples were labeled as follows: EC is Ebecryl containing $4 \mathrm{wt} . \%$ of cellulose nanocrystals, EC-ZN and EC-ZF are the nanocomposites containing 2 wt. $\%$ cellulose nanocrystals and $2 \mathrm{wt} . \% \mathrm{ZnO}$ nanostructures embedded in the acrylic resin. A second 
set of samples was prepared by depositing $600 \mathrm{~nm}$-thick AlN layer on the top surface of the previous ones (labelled $\mathrm{EC}+\mathrm{AlN}, \mathrm{EC}-\mathrm{ZN}+\mathrm{AlN}, \mathrm{EC}-\mathrm{ZF}+\mathrm{AlN}$ ) to investigate the change in the piezoelectric response. Piezoelectric characterization required the fabrication of $20 \times 15 \mathrm{~mm}^{2}$ rectangular cantilevers based on nanocomposite films, with and without an AlN layer. On both sides of the beams, $100 \mathrm{~nm}$-thick $\mathrm{Al}$ thin films were sputtered, working as electrodes. The devices were mounted on an electrodynamic shaker and the Al electrodes were connected to the DSO for recording the output signal, together with the signal of a reference accelerometer. They were tested at their resonance frequency and at three different accelerations (namely, 5, 7 and $10 \mathrm{~g}$ ). A square-shaped iron tip mass of $0.55 \mathrm{~g}$ with a surface area of $25 \mathrm{~mm}^{2}$ was added to the free end of all the cantilevers AlN-coated aiming at increasing the piezoelectric output voltage.

\section{Results and Discussion}

Piezoelectric characterization was performed on three beam configurations: the first one by dispersing $\mathrm{ZnO}$ nanostructures into EC matrix (characterized in its unfilled form, too), the second one adding a $600 \mathrm{~nm}$-thick AlN layer to the previous one and finally inserting a proof mass on the beam tip with the nitride layer. All the synthesized materials exhibit a piezoelectric response, confirming the compatibility of the explored matrix-filler combinations. The resonance frequency of the cantilevers with and without AlN layer was about $3.3 \mathrm{kHz}$. As expected, the proof mass addition at the beam tip shifts the resonance frequency toward a lower value $(3 \mathrm{kHz})$. The overall result, in terms of generated piezoelectric voltage for the three cases of study, is summarized in Figure 1.
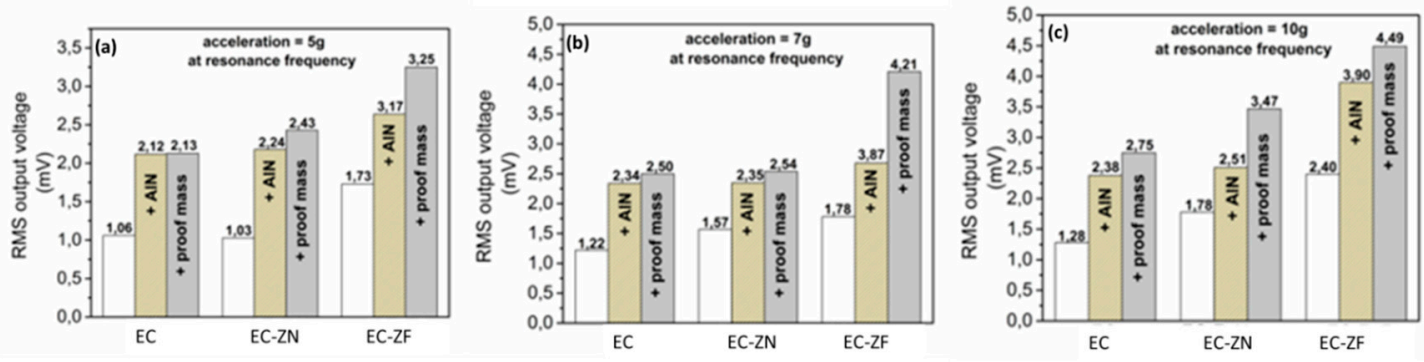

Figure 1. Root Mean Square output voltage values obtained by the different fabricated beams configurations at their resonance frequency for three acceleration values: (a) $5 \mathrm{~g}$, (b) $7 \mathrm{~g}$ and (c) $10 \mathrm{~g}$.

The Root Mean Square (RMS) output open circuit voltage, evaluated at three different accelerations at the resonance frequency, increases with the addition of the AlN layer on a surface of the beam and of a proof mass located at the tip of the device. In the first case, the piezoelectric contribution of AlN thin film can be taken into account, while the proof mass is responsible for a more effective applied strain. For all investigated cases, $\mathrm{ZnO}$ flower nanostructures provided the highest output signal.

\section{Conclusions}

Nanocomposite films were integrated as active material into cantilever-based generators and their piezoelectric response was investigated at different accelerations at the resonance frequency. All the devices exhibited a piezoelectric response that was improved by the addition of AlN layer as well as of a proof mass at the tip of the device. Flower-like structures exhibited the best piezoelectric performance. These findings suggest that the proposed UV-cured nanocomposites, having such a low fillers content, could be employed as potential energy harvesting thin coatings.

Author Contributions: M.A.S., C.D.P., D.D., G.M., L.F.: Writing-Original draft preparation. C.D.P. and L.F.: Software. G.M., A.F. and D.D.: Resources Investigation, Validation. L.F., G.M. and P.S.: Supervision. All authors have read and agreed to the published version of the manuscript.

Funding: This research received no external funding. 
Acknowledgments: The authors are grateful to E. Melissano, M. Masieri for their valid technician support.

Conflicts of Interest: The authors declare no conflict of interest.

\section{Reference}

1. Covaci, C.; Gontean, A. Piezoelectric Energy Harvesting Solutions: A Review. Sensors 2020, 20, e3512.

Publisher's Note: MDPI stays neutral with regard to jurisdictional claims in published maps and institutional affiliations.

(C) 2020 by the authors. Licensee MDPI, Basel, Switzerland. This article is an open access article distributed under the terms and conditions of the Creative Commons Attribution (CC BY) license (http://creativecommons.org/licenses/by/4.0/). 